\title{
Scheduling Mechanism for WLAN Frame Aggregation with Priority Support
}

\author{
Yang-Sheng Lin, Jun-Yao Wang ${ }^{1}$ and Wen-Shyang Hwang \\ Department of Electrical Engineering, National Kaohsiung University of Applied Sciences \\ ${ }^{1}$ Home Network Technology Center, Industrial Technology Research Institute / South
}

\begin{abstract}
For the overheads of 802.11 WLAN, several frame aggregation mechanisms had been proposed to deal with this shortcoming. Since the rare consideration of QoS in these proposals, we took advantage of $802.11 \mathrm{e}$ queueing model to enhance the priority scheduling. This paper approaches to eliminate the decomposition of aggregated frame to multi-destination. The idle while counting down the backoff time was been converted to advantage for frame scheduling in this paper.
\end{abstract}

Keywords: wireless LAN, frame aggregation, priority scheduling

\section{Introduction}

IEEE $802.11(\mathrm{Wi}-\mathrm{Fi})$ is the most popular wireless LAN environment nowadays. Its advantage of low price and easy deployment make it is undoubtedly the main extension for legacy wired LANs. However, due to multimedia applications' explosive growth, the requirements of high data rate are more desirable than it used to be in the past. Since September 2003, the Task Group N, an extension standard of IEEE 802.11 which plan to achieve a data rate higher than $100 \mathrm{Mbps}$, was begun. It aimed at solving the inefficient MAC of legacy IEEE 802.11 and proposed a new PHY mechanism, MIMO-OFDM. The prevailing approach of IEEE 802.11n MAC is to eliminate the overheads by introducing the Frame Aggregation mechanism.

These proposed mechanisms of frame aggregation are implemented at protocol layers include PHY, MAC, and even layers above MAC [2][4][5]. The general problem of these approaches is the tradeoff between efficiency and complexity. While the goal of this amendment is to provide a high speed environment for multimedia applications, the QoS support is necessary. According to this, the aggregation scheduling could be another research topic when consideration of priority is involved.

In this paper we proposed a frame aggregation mechanism which based on IEEE 802.11e. It could take advantage of backoff time to schedule frames. Frames which are destinated to the same destination are concatenated in a raw so as to eliminate the complexity of frame decomposition for multiple STA (Station). Additionally it remains the compatibility to standard IEEE 802.11e device and legacy Wi-Fi device.

The rest of this paper is arranged as follows. First we will give an introduction of QoS extension for IEEE 802.11 (Task Group E) and frame aggregation mechanisms. Then the detail about the mechanism we proposed will be given. Finally we will present some analysis of our proposed approaches and finish this paper with a conclusion.

\section{Background}

\subsection{IEEE 802.11e}

The queueing model of IEEE 802.11e is as shown in Fig.1, there are up to eight TCs (Traffic Category) could be implemented in a STA functioning as independent backoff instance. Each TCs is specified different PF (Persistent Factor) and AIFS (Arbitration Inter-frame Space). Referring to (1), Random() generates a pseudo-random number between 0 and $C W$ (Contention Window). $C W_{\text {MIN }}$ and $C W_{M A X}$ represent lower bound and upper bound of $C W$, respectively. $C W_{N E W}$ represents new $C W$ value for further collision. $C W_{\text {old }}$ represents the $C W$ size in last transmission Thus, by using (1), TCs could compute the $C W$ and get a random number of time slot for counting down and get into backoff state. The higher priority the TC is, the shorter the AIFS is.

$$
\begin{aligned}
& \text { BackoffTime }=\text { Random }() \times \text { SlotTime } \\
& C W_{N E W}=\left(C W_{\text {old }}+1\right) \times P F-1 \ldots \ldots \ldots \ldots . .
\end{aligned}
$$

The issue that we concern is: no matter how small the frame is, it permits only one frame for one TXOP (Transmission Opportunity) in IEEE 802.11e. Even the TC with high priority has more chance to be granted TXOP, the contention overhead also limits the performance and fairness. If the small frames in queue could be transmitted out faster, the frame in TC with lower priority could be granted TXOP earlier. According to this, it's feasible to apply frame aggregation into IEEE 802.11e for enhancement. 


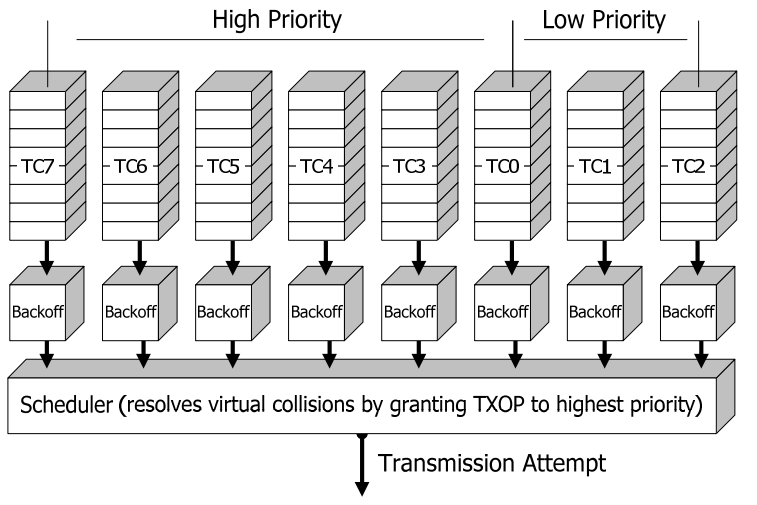

Fig.1: Traffic Categories in 802.11e

\subsection{Frame Aggregation Mechanisms}

The worst shortcoming of wireless LAN compare to wire network is low throughput performance. The reason is numerous overheads in MAC and PHY layers. These overheads include MAC header, Physical Layer Convergence Protocol (PLCP) preamble/header, acknowledgement (ACK) transmission, and some Inter Frame Spaces (IFSs). These overheads are added to each frame, thus seriously degrade the throughput performance of IEEE 802.11 wireless LAN. Even that IEEE 802.11 was approved in 1997, through out amendment of IEEE 802.11b, 802.11a and 802.11g, the MAC is remained unchanged till today. Accordingly, the emergency job to improve the throughput of IEEE 802.11 is a modification of MAC layer mechanism.

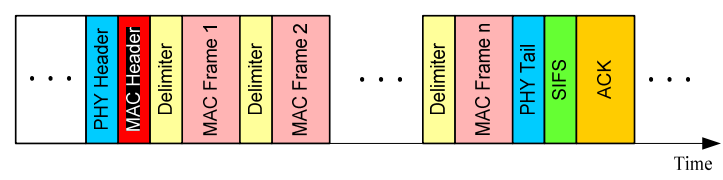

(a)

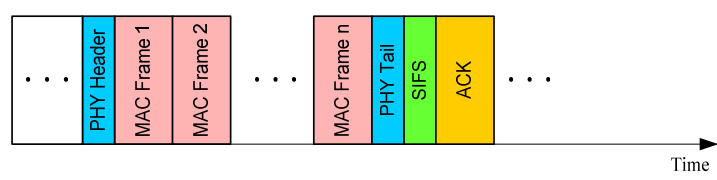

(b)

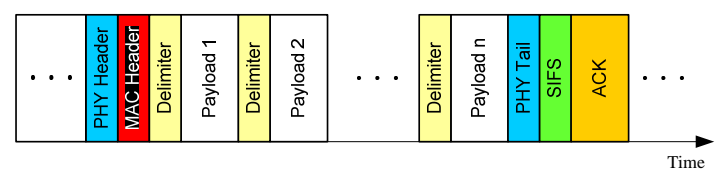

(c)

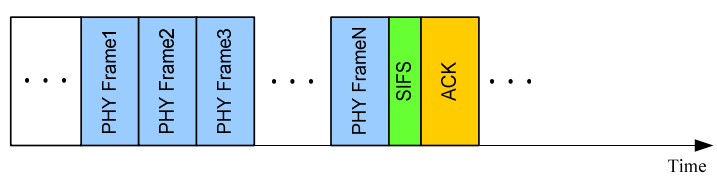

(d)
In most of proposals next generation wireless LAN, frame aggregation is a popular approach to eliminate the overhead of MAC layer of legacy IEEE 802.11 WLAN. The technique is to concatenate a number of frames into a single transmission unit, called virtual frame. Each virtual frame uses a MAC header, a PLCP, being transmitted in one transmission opportunity and being acknowledged by one ACK. Thus, for example, there are $\mathrm{N}$ frames which be aggregated into one virtual frame, the total overhead could be down to $1 / \mathrm{N}$. The benefit is more obvious in the environment crammed by small size frames. According to the report from Cooperative Association for Internet Data Analysis (CAIDA), the distribution of internet packet sizes is dominated by small size packets. Moreover the goal of IEEE $802.11 \mathrm{n}$ is to provide a network environment for multimedia application, which is full of small data units, the frame aggregation is definitely attractive for it.

According to Fig.2, there are several aspects of frame aggregation. The differences among these mechanisms include frame delimiting, method of acknowledgement, and the protocol layer at where the aggregation is implemented. When frames are distanced by delimiter, as shown in Fig.2(a), the aggregated frames can be distinguished by receiver clearly, but the aggregation is more inefficient. If these delimiters were removed as shown in Fig.2(b), the STA has to distinguish the frame boundary by MAC frame header. Such process will burden additional computation.

Aggregating frame at higher layer, as shown in Fig.2(c), will cause lower overhead of headers, but will also increase the complexity of decomposition of aggregated frames. When the frames are aggregated to different destination as shown in Fig.2(d), the least number of ACK is equal to the number of receiver. To make the number of ACK as less as possible, the frames destined to the same destination should be concatenated sequentially. By adopting block acknowledgement on each destination STA, the ACK could be down to the number of destination. Thus the overhead of sending one ACK to a frame could be saved, and the frame decomposition efficiency could also be increased.

In IEEE 802.11e, there is a mechanism, burst transmission, provided for STA to transmit a number of frames in a TXOP. While it provides an approach to save the contention time of each frame, there are still overheads in each transmitting frame. On the other hand, these frame aggregation mechanisms proposed by others rarely consider the QoS requirement in detail. This paper aimed at combination of IEEE 802.11e and frame aggregation mechanism to take the benefit of each other.

Fig. 2: frame aggregation 


\section{Mechanism}

We choose destination address as the queueing element instead of frame itself. As shown in Fig. 3, there is a pointer which points to a frame queue of a STA in each TC. When a TC is granted TXOP, it transmits the aggregated frame that is pointed by the pointer of the TC. After a successful transmission, the TC switches the pointer to another queue in the same TC. The decision mechanism of pointer switching could be random, the longest queue or other available scheduling approaches.

When a SDU is handed to MAC from upper layer, it is pushed into the queue according to its TC and destination address before aggregation and transmission. When the TC is granted TXOP, SDUs in the queue are aggregated into a big virtual frame and a header is attached in front of it. There are two time points that the SDUs should be aggregated. First, for VoIP, video stream and controlling data which have small size of frame, it could be aggregated until the TC is granted TXOP. The reason is that for small frames, there is less probability to exceed the aggregation threshold, which is usually equal to fragmentation threshold. Second, for frames that have relatively bigger size, it is necessary to aggregate the frame when the aggregated frame is expected to exceed the fragmentation threshold. The approach we used to prevent the oversize of aggregated frame is to break the pointer of linked-list of queue. As mentioned before, the implementation of queue is linked-list, every frame has a pointer which points to the next frame. The aggregation process is to aggregate the frames one-by-one according to the pointer. Because of this, we could easily build a boundary of aggregated frame by breaking the pointer when the queueing frames could make an oversize aggregated frame, and the aggregation process could work without consideration of frame size computation.

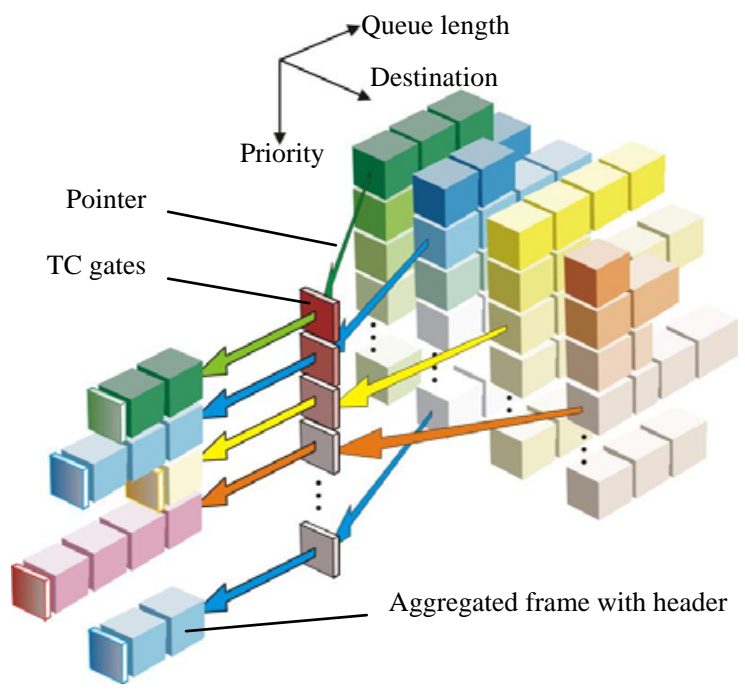

Fig. 3: Proposed mechanism model
Fig. 2 is the example of algorithm in pseudocode for determining the delimiter of aggregated frame.

$F_{\text {count }}:=$ size of aggregated frame

$F_{\text {thres }}:=$ fragmentation threshold

$F_{\text {data }}:=$ size of inputted frame

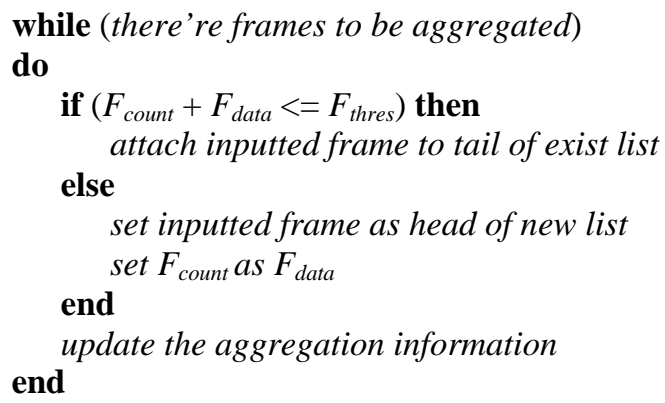

Fig.4: Algorithm of determining delimiter of aggregated frame

\section{Analysis}

In 802.11e, while the TCs have finished the contention process and start waiting for backoff time counting down, it is wasteful for these TCs to be just idle. Thus, leveraging the idle time for frame scheduling and aggregation is feasible. Actually, this duration is not limited in backoff time duration but extended to anytime as long as the STA has frame to send to certain STA. The frames are grouped by destination so as to aggregate frames with the same destination together in a block sequentially. This is also helpful while STA extracting the frames because it is ideal that STA can extract block of frame rather than inspect the frame one by one in a large aggregated frame. Additionally, it is helpful for STA to send an acknowledgement for whole block as soon as possible, and to increase the efficiency.

In other hand, while the frame aggregation mechanism rarely considers QoS, this paper proposed an amendment of frame aggregation which support frame classification before frames are aggregated. By adopting this mechanism, the aggregated frames will have the same attribute of destination and QoS priority. It is wonderful to combine the IEEE 802.11e with our frame aggregation mechanism. The composite mechanism has more dense computation and higher transmission efficiency. We discuss the performance in two aspects as follow.

\subsection{Throughput}

Consider the fixed overhead needed to transmit a data unit with DCF. These overhead include such as RTS/CTS/ACK frame, Inter-Frame spaces which is 
measured by time (ms/ $\mu \mathrm{s}$ ), and MAC header, PLCP header which measured by data (byte). We use $O_{T}$ and $O_{D}$ to represent the overhead of time and overhead of data respectively. Thus we have (3) for representing the throughput performance of one transmission opportunity.

$$
\frac{n \cdot O_{D}+N \cdot S D U}{n \cdot O_{T}+N \cdot T}
$$

$S D U$ is the data to be transmitted, which could a single frame or a relative bigger virtual frame which is aggregated by number of frames (or payload). $T$ is the transmission time of PDU which is equal to the dividend. $N$ is the number of overhead for both data and time, and $N$ is the number of frame to be aggregated.

Without frame aggregation, $n$ is equal to $N$. This means that no matter how big $N$ is, the throughput is nearly invariable. In contrast, $n$ and $N$ is independent to each other while using frame aggregation mechanism. Because several frames are transmitted at a time, there is only one set of overhead needed. Thus $n$ is equal to 1 , and $N$ is equal to the number of frame which are aggregated. We could modify (3) to form the equation shown in (4).

$$
\frac{O_{D}+N \cdot S D U}{O_{T}+N \cdot T}
$$

By comparing (3) and (4), it is clear that frame aggregation mechanism has higher throughput performance than legacy one due to $N$ is always greater than or equal to 1 .

\subsection{Fairness}

There are two problems which may affect the fairness and needed to be solved in this mechanism. The first one is which TC to be granted TXOP. This job is already standardized in IEEE 802.11e EDCF mechanism; hence we don't want to pay attention on it. The other problem is which destination should be scheduled for next transmission in a TC. Considering a scenario, there is a application such as VoIP or VOD which continually generating high priority data to a certain STA. If the other applications were generating normal priority data, there is a high probability that these applications could suffer a starvation. To avoid the situation, we involved two parameters $Q L_{i}$ and D-last ${ }_{i}$. $Q L_{i}$ represents the queue length of destination $i$, and $D$ last $_{i}$ represents the sum of data transmitted to destination $i$ in last transmission. Referring to (5) :

$$
\operatorname{MAX}\left\{\frac{1}{D-\text { last }_{i}} \cdot Q L_{i}, i=\left[1, N_{\text {dest }}\right]\right\}
$$

In (5), $N_{\text {dest }}$ represents the number of destination. Thus, the destination which has the maximum value could be found by (5), and the destination for next transmission in a TC could be decided.

\section{Conclusion}

Frame aggregation mechanism is an important part of MAC enhancement for next generation WLAN. It adopts several approaches that concatenate a number of frames into a large frame so as to eliminate overheads include ACK and interframe space, thus increase the transmission efficiency. For small frames like network control or multimedia application data, frame aggregation is a favorable method, but QoS also take an important role herein. This paper proposed a enhancement mechanism for WLAN base on 802.11e and frame aggregation. By adopting this mechanism, STA could aggregate frame more graceful and efficient.

\section{References}

[1] Y. Xiao and J. Rosdahl, “Throughput and Delay Limits of IEEE 802.11,” IEEE Commun. Lett., vol. 6, no. 8, Aug. 2002, pp.355-57.

[2] Y. Kim, S. Choi, K. Jang, H. Hwang, "Throughput Enhancement of IEEE 802.11 WLAN via Frame Aggregation,” Vehicular Technology Conference., vol. 4, Sept. 2004, pp.3030-3034.

[3] Yang Xiao, "Packing Mechanisms for the IEEE 802.11n Wireless LANs,” Global Telecom. Conference, vol. 5, 29 Nov.-3 Dec. 2004, pp.3275-3279.

[4] C. Liu and A. P Stephens, "An Analytic Model for Infrastructure WLAN capacity with Bidirectional Frame Aggregation," Wireless Comm. and Networking Conf., vol. 1, 13-17 Mar. 2005, pp.113-119.

[5] Yang Xiao, "IEEE 802.11n: Enhancements for Higher Throughput in Wireless LANs,” IEEE Wireless Communications, vol. 12, no. 6, Dec. 2005, pp.82-91.

[6] Yang Xiao, “IEEE 802.11 Performance Enhancement via Concatenation and Piggyback Mechanisms," IEEE Tranc. on Wireless Comm., Vol. 4, no. 5, Sept. 2005, pp.2182-2192.

[7] S. Mangold, S. Choi, P. May, O. Klein, G. Hiertz, and L. Stibor. "IEEE 802.11e wireless LAN for quality of service”, Proc. European Wireless, 2002. 